\title{
Imaging features of spinal fractures in ankylosing spondylitis and the diagnostic value of different imaging methods
}

\author{
Cui Ren, Qiao Zhu, Huishu Yuan \\ Department of Radiology, Peking University Third Hospital, Beijing, China \\ Correspondence to: Huishu Yuan. Department of Radiology, Peking University Third Hospital, 49 North Garden Road, Haidian District, Beijing \\ 100191, China. Email: huishuy@bjmu.edu.cn.
}

Background: Our study aimed to characterize the imaging appearance of spinal fractures in ankylosing spondylitis (AS) and identify situations in which the use of magnetic resonance imaging (MRI) is necessary.

Methods: A total of 70 cases of spinal fractures associated with AS were retrospectively enrolled. Two radiologists independently reviewed the preoperative images. The location, type, ligament injury, neurological injury, and epidural hematoma following spinal fractures were assessed.

Results: Only one patient had a vertebral compression fracture, and 69 patients had 77 transverse fractures involving three columns. The most frequent injuries in AS patients were type B3 (N=32, 43.8\%) spine fractures, followed by type $\mathrm{C}(\mathrm{N}=20,27.4 \%)$ spine fractures. There were significant differences in fracture types of the different spine regions $(\mathrm{H}=14.1, \mathrm{P}<0.0001)$. Most type $\mathrm{C}$ spine fractures were located in the lower cervical spine, while most of the type B2 spine fractures were located in the thoracic spine. Transverse fractures were classified as shear or stress type fractures. In total, there were 62 shear fractures and 15 stress fractures. All of the transverse fractures were detected by computed tomography (CT). The accuracy of CT in the diagnosis of the exact anatomic involvement of transverse fractures was significantly higher than that of MRI $\left(\chi^{2}=8.36, \mathrm{P}=0.014\right)$. The anterior longitudinal ligament (ALL) was the most frequently torn ligament. Tears of ossified ligaments were best visualized by sagittal reformatted CT. Lower cervical fractures were more likely to be associated with neurological injury compared with fractures to other regions of the spine $\left(\chi^{2}=7.24, \mathrm{P}=0.025\right)$. There were six epidural hematoma cases, which were only detected by MRI, were found to have fractures of the lower cervical spine.

Conclusions: We recommend a whole-spine CT examination with three-dimensional reconstruction for detecting a suspected fracture in AS patients. In cases with neurological injury, MRI examinations are always mandatory. AS patients with lower cervical spine fractures require further investigation by MRI. Patients with non-lower cervical spine fractures without any neurological deficits do not need to undergo an immediate MRI.

Keywords: Ankylosing spondylitis (AS); spinal fractures; radiography; computed tomography (CT); magnetic resonance imaging (MRI)

Submitted Aug 10, 2020. Accepted for publication Feb 05, 2021.

doi: 10.21037/qims-20-962

View this article at: http://dx.doi.org/10.21037/qims-20-962

\section{Introduction}

Ankylosing spondylitis (AS) is a chronic inflammatory disease with primary axial skeleton involvement. The main pathological changes include ossification of ligaments, disc annuli, and facet joints, which ultimately leads to spinal rigidity and changes to the spine's biomechanical stability. Also, AS is accompanied by varying degrees of osteopenia or osteoporosis, which further reduces the spine's stability, and even a minimal force can cause spinal fracture $(1,2)$. 
The incidence of spinal fracture in patients with AS is $7-8$ times higher than in normal subjects $(3,4)$. AS patients with spinal fracture characteristics differ from other patients, and fractures often involve three columns of the spine and are more easily associated with a dislocation. The incidence of nerve injury is also significantly higher than after a general spinal fracture $(5,6)$. The continuous motion of fracture segments is accompanied by secondary complications, such as functional pseudoarthrosis and aggravation of nerve damage. Early diagnosis and proper treatment are critical.

By recognizing all fractures' imaging features and fully characterizing the extent of the injury, a radiologist can reduce the risk of a missed or delayed diagnosis of an injured ankylosing spine. The cohort size has limited previous imaging studies on spinal fractures in AS patients. AS spinal fractures can be easily missed on radiographs, and therefore, CT examination is recommended. The particular value of MRI over CT is the evaluation of neurological and disco-ligamentous injuries. However, MRI can cause further neurological injury due to positioning. It is also technically challenging to obtain MR images of good diagnostic quality in traumatized patients with advanced AS, and severe kyphosis of the thoracic spine can cause difficulties among AS patients in lying supine or even fitting into a closedbore MR scanner $(7,8)$. MRI and CT are complementary modalities for the detection of fractures in patients with AS. Although whether MRI examinations are mandatory in spinal trauma patients with AS and whether MRI is more useful than CT in the detection of additional injuries influencing the selection of treatment methods remains unknown. Therefore, this study aimed to explore the imaging characteristics of spinal fracture in AS patients and assess the diagnostic value of different imaging methods.

\section{Methods}

\section{Subjects}

This retrospective study was approved by the Institutional Ethics Committee, and the requirement for obtaining written informed consent was waived. Patients were identified by searching spinal radiograph, CT, and MRI reports from January 2012 to January 2020 in our Picture Archiving and Communication Systems (PACS) database for the following keywords: AS and fracture. All of the patients were diagnosed with AS before the injury, and imaging findings were consistent with AS. All patients fulfilled the American College of Rheumatology (1984 revised) AS diagnostic criteria. We excluded three patients with poor image quality. Seventy AS patients comprising 60 males and 10 females with spinal fracture were finally enrolled in our study, with a mean age of 53.52 years (standard deviation $=13.45$, range $22-82$ years). All subjects underwent preoperative imaging examination.

\section{Imaging examination}

Radiography consisted of anteroposterior and lateral views, and digital images were obtained by GE Discovery XR650 (GE Healthcare, America). For CT examination, Siemens Somatom Definition Flash dual-source CT (Siemens Healthcare, Germany) or GE Discovery CT750 HD CT was used, and the parameters were as follows: slice thickness, $1 \mathrm{~mm}$; interval, $1 \mathrm{~mm}$. For multi-planar reformatted images, the parameters were as follows: slice thickness, $3 \mathrm{~mm}$; interval, $3 \mathrm{~mm}$, and the bone plus soft tissue algorithm. For MRI examination, Siemens Trio 3.0-T MR or GE Discovery MR750 3.0-T MR was used. The following MR protocol was applied: sagittal T1-weighted fast spin-echo (FSE) sequences, sagittal T2-weighted FSE sequences, sagittal fatsuppressed (FS) T2-weighted FSE sequences, and axial T2weighted FSE sequences. All of the MR images were 3 or $4 \mathrm{~mm}$ thick with a $0.4-$ or $0.5-\mathrm{mm}$ gap.

\section{Image evaluation}

A fracture line on radiographs or CT images was defined as the presence of a sharp lucent or sclerotic line within the trabecular bone, and a fracture line on MR images was defined as a linear or curvilinear focus of low signal intensity on T1WI that showed either low or high intensity on FS-T2WI (9). Other criteria for fracture included a break in the continuity of the cortex or a dislocation of bone fragments (10). Evidence of a zone of diffusely increased signal intensity on FS-T2WI was interpreted as bone marrow edema but not as a manifestation of fracture. The occult fracture was defined as a fracture that was not visible on plain radiographs but it was identifiable on other imaging modalities.

A junior radiologist and a senior radiologist separately interpreted the spinal radiography, CT, and MRI examinations. Three reading sessions were performed with an interval of 4 weeks. In each session, only one imaging modality was evaluated without knowledge of the other two imaging findings. Two observers evaluated only the levels of 


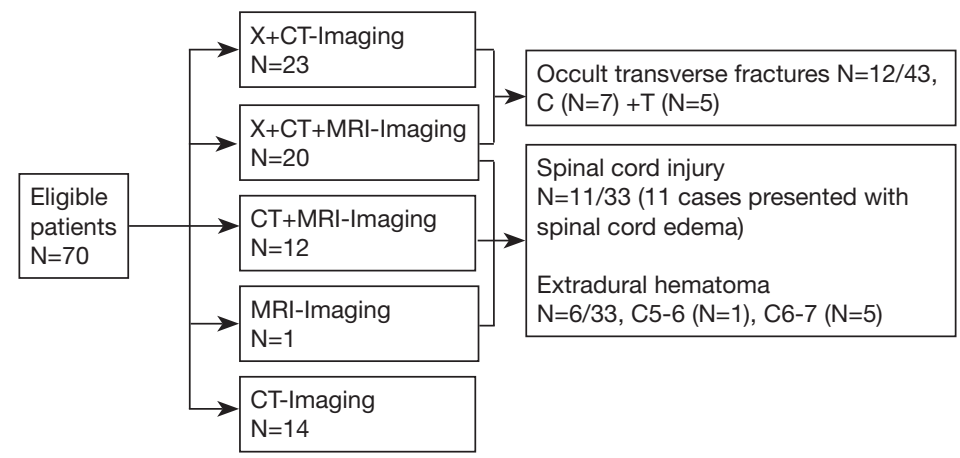

Figure 1 Flowchart illustrating the data analysis of spinal fracture in patients with AS. AS, ankylosing spondylitis; N, number of patients; C, cervical spine; T, thoracic spine.

spinal fractures on radiography; however, they recorded the exact anatomic involvement of fractures on CT and MRI. Ligament tears, spinal cord injury, and epidural hematoma were also assessed.

Intraoperative findings were used as the reference standard; however, not all of the spinal structures were visualized during each surgery, depending on the surgical approach. The final diagnosis or structure of involvement was established by consensus among the three authors. Any disagreement was resolved through discussion among all authors and with a senior spine surgeon's involvement until a consensus was reached.

\section{Statistical analysis}

SPSS18.0 software (SPSS, Chicago, IL, USA) was used for statistical analysis. Furthermore, the diagnosis results of the senior doctor were also used for statistical analysis. Fracture type differences among the different regions of the spine were evaluated by the Kruskal-Wallis $\mathrm{H}$ test. The accuracy of CT and MRI in diagnosing the exact anatomic involvement of transverse fractures was compared using the Chi-square test. Neurological injuries among the different regions of the spine were also evaluated with the Chi-square test. A P value $<0.05$ indicated a statistically significant difference for all tests.

\section{Results}

AS patients with acute spine fractures $(\mathrm{N}=47)$ underwent imaging examination within 0 to 20 days after injury, and patients with chronic spine fractures $(\mathrm{N}=23)$ were referred for imaging at least 1 month after injury. The intervals between preoperative imaging and surgery were between 1 and 7 days. Among the 70 included patients, 43 cases underwent X-ray and MRI /CT examinations to analyze occult fracture, and 33 patients underwent MRI examination for the analysis of spinal cord injury and epidural hematoma. A total of 12 cases of occult transverse fractures were located in the cervical and thoracic spine. Moreover, a total of 11 cases of spinal cord injury and six cases of epidural hematoma were found to have transverse fractures that were only detected by MRI (Figures 1,2).

\section{Surgical treatment strategy}

Of the 30 patients with cervical fractures, one patient with C1-2 fractures had undergone anterior surgery with decompression, fusion, and plating; and 11 patients had undergone anterior surgery combined with posterior stabilization with pedicle and/or lateral mass screw fixation. The remaining 18 patients with cervical fractures were treated with posterior reduction, decompression, and instrumented fusion with pedicle or lateral mass screw fixation (or a combination of both). All 39 patients with thoracic/lumbar fractures or multiple fractures had undergone posterior surgery with reduction, decompression, and internal fixation with pedicle and/ or lateral mass screw fixation, except for one patient with multiple fractures who had undergone anterior cervical and posterior thoracolumbar surgery.

\section{Fracture characteristics}

Of the 70 included patients, 69 patients had transverse fractures involving three columns of the spine with or without compression or appendix fractures that were not part of transverse fractures, while the remaining patient 

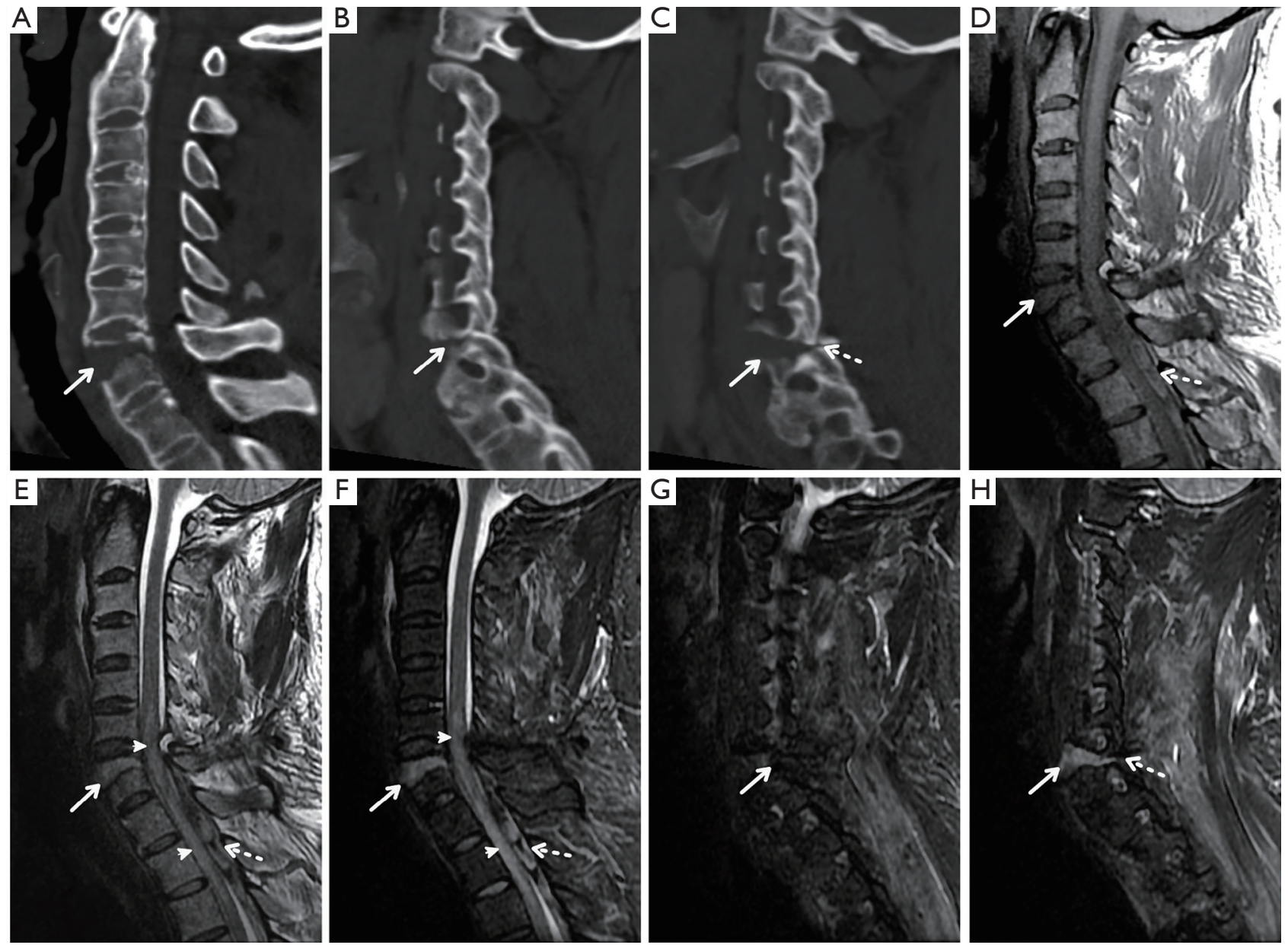

Figure 2 Spinal fracture of a 52-year-old man with AS. Midline sagittal CT (A) and paramedian sagittal CT (B and C) demonstrated a fracture traversing the $\mathrm{C} 7$ vertebral body (solid arrow), bilateral pedicles (solid arrow), and right articular process (dashed arrow). Disruption of the ossified anterior longitudinal ligament (solid arrow) at the level of the fracture was also detected. Midline sagittal T1WI (D), T2WI (E), and FS-T2WI (F) demonstrated the displacement of fracture segments and no obvious marrow edema adjacent to the fracture site in C7 (solid arrow). On the same slice as CT, paramedian sagittal FS-T2WI (G and H) could not clearly show the range of the fracture line. High signal intensity edema (arrowheads) in the spinal cord indicated medullary contusion, and dorsal epidural hematoma at C7-T3 level was also found (dashed arrow). AS, ankylosing spondylitis.

had an L2 compression fracture. A total of 63 patients had a single transverse fracture, and six patients had 14 multiple transverse fractures. The majority of single fractures were located in the cervical spine $(\mathrm{N}=30,38.5 \%)$, and the most commonly affected segment was C6-7 ( N=15). The thoracic spine was involved in $23(29.5 \%)$ cases, and the most commonly affected segment was T11-12 (N=9). The lumbar spine was involved in 11 (14.1\%) cases, and L1-2 $(\mathrm{N}=5)$ was the most commonly affected segment (Table 1).

The types of fractures (except for upper cervical fractures) were classified using the Arbeitsgemeinschaft für
Osteosynthesefragen (AO) classification. The most frequent injuries were type $\mathrm{B} 3(\mathrm{~N}=32,43.8 \%)$ spine fractures, followed by type $\mathrm{C}(\mathrm{N}=20,27.4 \%)$ spine fractures (Table 2). There were significant differences in fracture types among the different spine regions $(\mathrm{H}=14.1, \mathrm{P}<0.0001)$. Pairwise comparisons revealed significant differences between the cervical and thoracic spine $(\mathrm{H}=3.74, \mathrm{P}<0.0001)$. Most type $\mathrm{C}$ spine fractures were located in the cervical spine, while most of the type B2 spine fractures were located in the thoracic spine.

The types of transverse fractures were classified as 
Table 1 Distribution of spinal fractures in patients with ankylosing spondylitis

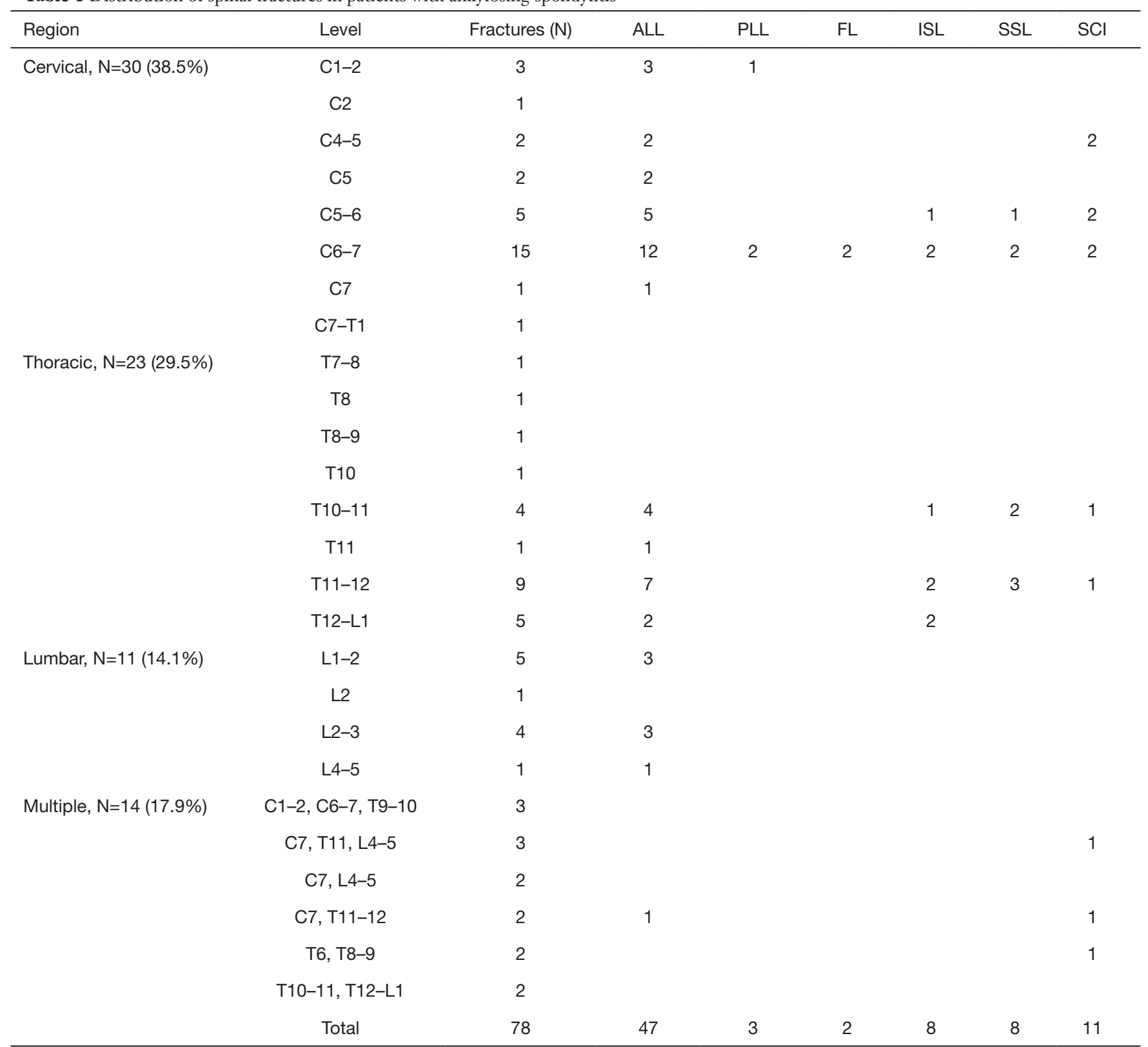

ALL, tearing of the anterior longitudinal ligament; PLL, tearing of the posterior longitudinal ligament; FL, tearing of the ligamentum flavum; ISL, tearing of the interspinous ligament; SSL, tearing of the supraspinous ligament; SCI, spinal cord injury

either shear or stress fractures. In total, there were 62 shear fractures and 15 stress fractures. Furthermore, 31 cases of shear fractures occurred in the cervical spine, which was the most common site. All of the stress fractures were located in the thoracic and lumbar spine, except for one case, which was located in the C6-7 segment, and the thoracolumbar spine was the most common site.
There were four types of transverse fractures through or not through the pedicle: single vertebral body with the same or adjacent segmental appendix fractures in 15 cases (Figure $3 A, B$ ), two adjacent vertebral bodies with fused apophyseal joint fractures in three cases (Figure 3C), single vertebral body with the same level fused apophyseal joint fractures in 38 cases (Figure 3D), and trans-discal fractures 
Table 2 AO classification of AS spinal fractures

\begin{tabular}{lccccc}
\hline Region & A & B1 & B2 & B3 & C \\
\hline Cervical & 0 & 0 & 3 & 14 & 12 \\
Thoracic & 0 & 1 & 14 & 6 & 3 \\
Lumbar & 1 & 0 & 2 & 32 & 20 \\
Total & 1 & 1 & 19 & 4 \\
\hline
\end{tabular}

AO, Arbeitsgemeinschaft für Osteosynthesefragen; AS, ankylosing spondylitis.
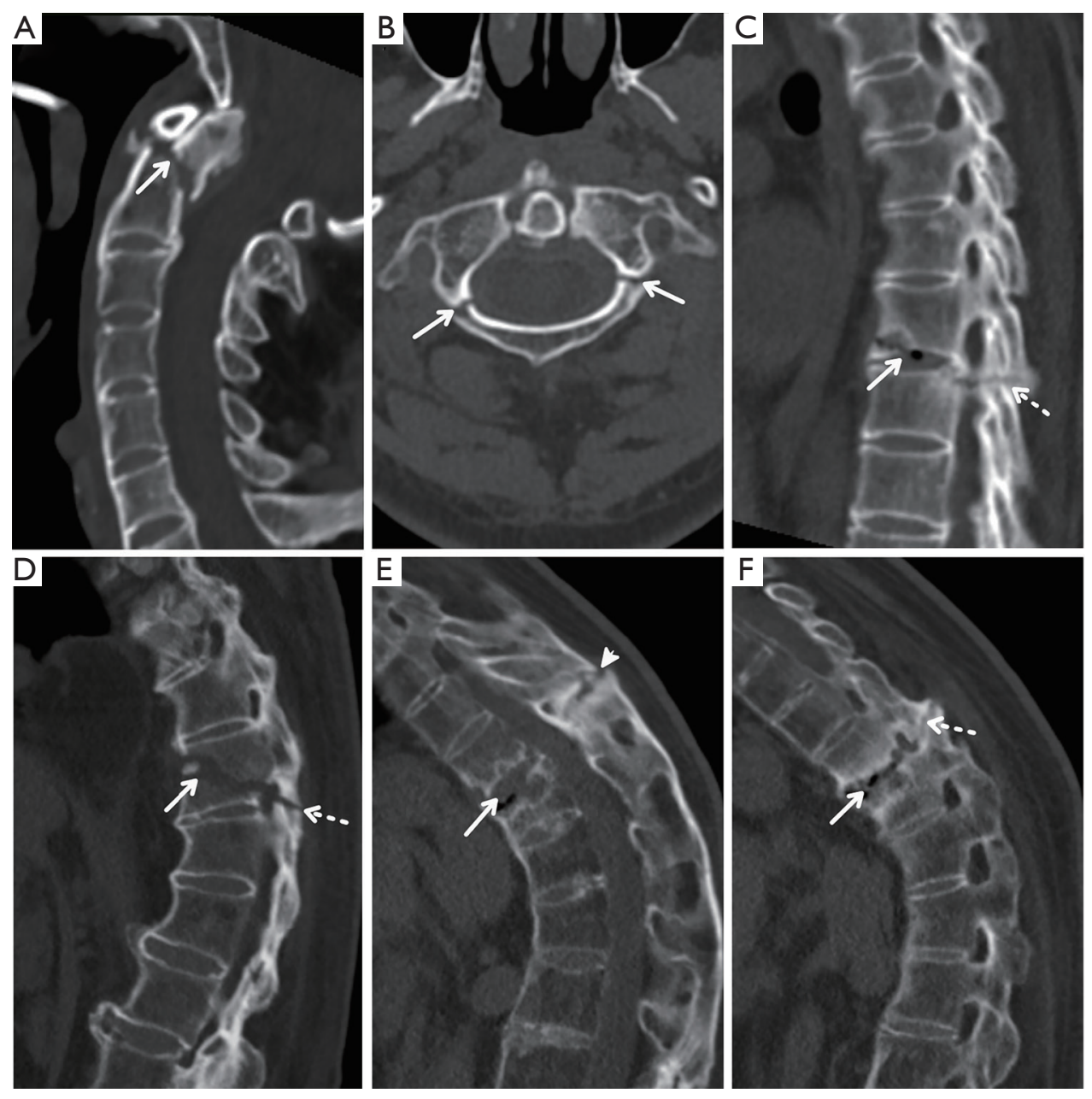

Figure 3 Four types of transverse fractures in AS patients. Midline sagittal CT (A) and axial CT (B) of the cervical spine in a 36-year-old man with AS showed an odontoid process fracture (solid arrow) and a variant Jefferson fracture (solid arrow). Paramedian sagittal CT (C) of the thoracic spine in a 62-year-old female with AS showed that the fracture line was located in the anterior inferior border of T7 and the posterior superior border of T8 (solid arrow), involving fused apophyseal joint of T7 and T8 (dashed arrow). Sagittal CT (D) of the thoracic spine in an 82-year-old male with AS showed a vertebral fracture at T11 (solid arrow) involving fused apophyseal joint of T11 and T12 (dashed arrow). Sagittal CT images of (E and F) in a 48-year-old male with AS showed T11-T12 trans-discal destruction, erosion, and reactive sclerosis in adjoining vertebral bodies (solid arrow) with the trans-discal fracture extending to the fused apophyseal joint of T11 and T12 (dashed arrow), as well as a tear of the ossified supraspinous ligament (arrowhead). AS, ankylosing spondylitis. 
Table 3 Neurological injury by ASIA spinal injury grade in patients with ankylosing spondylitis

\begin{tabular}{lllllll}
\hline Region & A & B & C & D & A-D & E \\
\hline Cervical & 8 & 2 & 2 & 10 & 22 & 11 \\
Thoracic & 1 & 2 & 0 & 5 & 8 & 17 \\
Lumbar & 0 & 0 & 0 & 5 & 5 & 7 \\
Total & 9 & 4 & 2 & 20 & 35 & 35 \\
\hline
\end{tabular}

All cervical fractures with neurological injury were located in the lower cervical spine. ASIA, American Spinal Injury Association.

with the same level fused apophyseal joint fractures in 21 cases (Figure 3E,F).

A total of 32 patients with 37 transverse fractures underwent preoperative CT and MRI examinations. The intervals between CT and MRI scans were 0-3 days. CT accuracy in diagnosing the exact anatomic involvement of transverse fractures was significantly higher than that of MRI $\left(\chi^{2}=8.36, P=0.014\right)$. CT showed the exact anatomic involvement of transverse shear fractures more clearly than MRI. CT missed three cases of acute non-displaced spinous process fracture that were not part of the transverse fractures. However, these stable fractures did not affect the treatment plan.

\section{Ligament rupture}

Rupture of the ossified ligaments was best visualized by sagittal reformatted CT, which included 47 cases of anterior longitudinal ligament (ALL) rupture, five cases of supraspinal ligament rupture, and four cases of interspinous ligament rupture. Rupture of the non-ossified ligaments was only depicted by MRI, which included two ligamentum flavum rupture cases, four cases of interspinous ligament rupture, and three cases of posterior longitudinal and supraspinal ligament rupture confirmed by surgical examination. Two cases of interspinous ligament integrity evaluated by MRI were coincident with their surgical findings. One case of interspinous ligament rupture confirmed by surgery showed no obvious abnormal signals on MRI, and one case of interspinous ligament edema on MRI was correlated surgically with the intact ligament.

\section{Neurological injury}

Neurological deficits of AS patients were preoperatively assessed using the American Spinal Injury Association (ASIA) Classification System. In total, 35 of 70 patients
(50.0\%) had neurological injuries (ASIA grade: A-D). There were significant differences in fractures of different spine regions that were complicated with neurological deficits $\left(\chi^{2}=7.24, \mathrm{P}=0.025\right)$. Lower cervical fractures were more likely to be associated with neurological injury compared with fractures in other regions of the spine (Table 3).

\section{Discussion}

AS patients are susceptible to spinal fractures even after a low-velocity impact or injury, and fractures might not be noticeable due to long-standing inflammatory back pain. Early diagnosis and treatment of AS patients with a clinically suspected spinal fracture can minimize the risk of complications and prevent unnecessary neurological deterioration.

Hyperextension or hyperflexion has been considered as the leading trauma mechanism in ankylosing spinal diseases (11). In our study, the most frequent injuries were type B3 fractures according to the AO classification. Spinal fractures in AS on imaging can also be roughly divided into three types: shear fracture, stress fracture (or pseudoarthrosis), and vertebral compression fracture. Due to altered spinal biomechanical properties, AS spinal fractures often involve three columns of the spine; however, free bone fragments in the spinal canal are rare. In our study, spinal fractures, including shear fractures, were most likely to occur at the C6-7 level, which was in line with previous reports (12-14). The lower cervical spine is the most commonly injured area because of increased mobility, small vertebral bodies, oblique articular facets, and the skull's mobility (1). In this study, most stress fractures occurred in the thoracolumbar segment. The kyphosis apex of the normal spine is located in T7; however, the kyphotic apex of AS patients gradually moves to the thoracolumbar junction (T10-L2). Their fixed and kyphotic spine's apical region is subjected to the highest level of 
stress (15). A stress fracture is characterized by the presence of extensively destructive disc-vertebral lesions and sclerosis of the adjacent bone and was first described by Andersson in 1937 (16). However, whether traumatic or inflammatory factors have a greater impact on the development of extensive Andersson lesions remains unclear.

Despite the many advantages of CT, radiography remains the first-line imaging modality to detect or exclude the presence of a fracture (17). Diagnosis of spinal fractures in AS by radiography alone can be challenging. Our results showed that all occult transverse fractures were located in the cervical and thoracic spine, which is consistent with previous studies $(5,6)$. There are several possible explanations for this: spinal kyphotic deformity, ossification of the spinal ligaments, disc spaces are poorly outlined, osteoporosis, and detection of fractures located in the lower cervical spine and the cervical-thoracic junction is difficult due to overlapping of the shoulder girdle $(12,18-20)$. Radiographs are poor at depicting fractures of the rigid spine, and multiple noncontiguous fractures have been reported in $6-8 \%$ of cases (21). Six of the 70 included patients were found to have multi-segment or multilevel transverse fractures in our series. Also, spinal fracture in AS patients might not be noticeable; a previous study reported that minor trauma could lead to spinal fractures, where minor trauma included the impact that many might not describe as 'trauma,' such as a history of driving over rough ground or a cough (22), and thus, some patients may have no definite history of trauma. Therefore, whole spine CT is recommended for fracture screening in AS patients following an injury. Additionally, CT has the advantage of a shorter scanning time, as well as excellent spatial resolution and multiplanar reconstruction, and can precisely depict the extent of acute fractures.

However, the more suitable imaging modality for recognizing spinal fractures in AS patients (CT or MRI) remains an open question. In the current study, CT missed three non-displaced spinous process fractures that were not part of the transverse fractures. However, these stable fractures on CT examination did not affect the treatment plan. We found that the anatomic involvement of spinal transverse shear fractures was more clearly seen on CT compared to MRI. Previous studies revealed the superiority of CT over plain radiographs or MRI in showing detailed information on fracture morphology; although, MRI can detect some injuries and identify some additional fractures that are undetectable by CT $(7,8)$. Fractures that distract or do not show compression on CT do not reliably generate marrow edema on MRI and could lead to a false-negative MRI; additionally, there is a paucity of marrow edema noted in the setting of spinal ankylosis (23). A similar result was noted in our study; very little or no marrow edema was detected by MRI scanning in some acute fracture areas confirmed by CT. Bone marrow edema is an important non-specific sign for detecting non-displaced shear fractures on MRI. However, diffuse marrow edema can affect the judgment of the extent of a fracture line.

MRI is particularly valuable in showing neurological, disco-ligamentous, and soft tissue injuries. The prevalence of spinal cord injury in AS patients is more than 11 times greater than in the general population (3). Several studies have shown a markedly higher prevalence of neurological complications ranging from $33 \%$ to $58 \%$ for thoracic and lumbar fractures and even higher cervical spine rates (24). Spinal cord injuries may manifest as spinal cord contusion, hematoma, transection, cord compression due to fracture, or epidural hematoma.

AS patients have a higher incidence of extradural hematoma (SEH) than the general population (25). SEH should be considered in cases of sudden and rapid neurological deterioration and has been noted in $21.6 \%$ of AS patients (26). In our study, epidural hematoma incidence was $18.1 \%$, which was not diagnosed by CT. Only C5-7 transverse fracture combined with SEH was identified. SEH is mostly located on the dorsal side and typically extends over multiple levels. It is unknown why patients with AS are prone to have a higher risk for epidural hematoma after spinal trauma. One theory is that the stiffer spine is accompanied by less elastic paraspinal vasculature that can rupture easily. Also, AS patients sustain fractures typically with a transverse extension distraction mechanism, and the paraspinal vasculature can easily rupture with this highly unstable injury pattern (27).

Ossified ALL tears can be identified on radiographs, except for occult fractures; however, tears to the posterior longitudinal ligament (PLL) and ligaments in the posterior column cannot be delineated. Ossified ligament tears are best visualized by sagittal reformatted CT, while non-ossified ligamentous disruption is not identifiable on CT. Disruption of the normal vertebral alignment may be an indicator of PLL disruption. MRI is ideal for the evaluation of nonossified ligamentous injuries, and T2WI with fat suppression is more sensitive in detecting edema than other sequences. Ligamentous partial tears on fat-suppressed T2WI appear as a slightly or markedly increased signal within the ligament with varying degrees of intact fibers, while complete tears of 
the ligament appear as a markedly high signal intensity with complete disruption in the continuity of the fibers. Variable performance of MR for ligamentous injury has been noted in different studies and compared with surgical exploration and direct evaluation of the ligamentous structure, with different investigators reporting good, moderate, or poor correlations (28-30). In our cohort, one case of interspinous ligament rupture confirmed by surgery showed no obvious abnormal signals on MRI, and one case of interspinous ligament rupture showed high signals on MRI and was confirmed as not having rupture during surgery. An MRI scan was performed at a stage of no edema or hemorrhage collection in ruptured ligaments. Also, soft-tissue edema near the ligaments could affect the observation of ligaments themselves, explaining our results.

Our study has several limitations that should be noted, including the inherent limitations of retrospective research and the fact that not all patients underwent spinal $\mathrm{X}$-rays, CT, and MRI examination at the same time before surgery, which may be related to the clinical symptoms of the patients and clinicians' preferences for imaging. Therefore, we cannot rule out the possibility of selection bias in the present study. Also, some spinal injuries may have been missed due to different surgical approaches. Lastly, the inter-observer agreement of the exact anatomic involvement of fractures and ligaments disruption was not assessed.

\section{Conclusions}

Based on our study results, we recommend a whole-spine CT examination with three-dimensional reconstruction for detecting a suspected fracture in AS patients in the first instance wherever possible. In cases with neurological injury, MRI examinations are always mandatory. Furthermore, AS patients with lower cervical spine fractures, which have a higher incidence of neurological injury and epidural hematoma, require further investigation by MRI. Patients with non-lower cervical spine fractures without any neurological deficits do not need to undergo an immediate MRI.

\section{Acknowledgments}

Funding: This study was supported by the National Scientific Foundation of China (No. 81871326).

\section{Footnote}

Conflicts of Interest: All authors have completed the ICMJE uniform disclosure form (available at http://dx.doi. org/10.21037/qims-20-962). The authors have no conflicts of interest to declare.

Etbical Statement: This retrospective study was approved by the Institutional Ethics Committee, and the requirement for obtaining written informed consent was waived.

Open Access Statement: This is an Open Access article distributed in accordance with the Creative Commons Attribution-NonCommercial-NoDerivs 4.0 International License (CC BY-NC-ND 4.0), which permits the noncommercial replication and distribution of the article with the strict proviso that no changes or edits are made and the original work is properly cited (including links to both the formal publication through the relevant DOI and the license). See: https://creativecommons.org/licenses/by-nc-nd/4.0/.

\section{References}

1. Leone A, Marino M, Dell'Atti C, Zecchi V, Magarelli N, Colosimo C. Spinal fractures in patients with ankylosing spondylitis. Rheumatol Int 2016;36:1335-46.

2. Vosse D, Feldtkeller E, Erlendsson J, Geusens P, van der Linden S. Clinical vertebral fractures in patients with ankylosing spondylitis. J Rheumatol 2004;31:1981-5.

3. Shah NG, Keraliya A, Nunez DB, Schoenfeld A, Harris MB, Bono CM, Khurana B. Injuries to the Rigid Spine: What the Spine Surgeon Wants to Know. Radiographics 2019;39:449-66.

4. Rustagi T, Drazin D, Oner C, York J, Schroeder GD, Vaccaro AR, Oskouian RJ, Chapman JR.Fractures in Spinal Ankylosing Disorders: A Narrative Review of Disease and Injury Types, Treatment Techniques, and Outcomes. J Orthop Trauma 2017;31:S57-S74.

5. Westerveld LA, van Bemmel JC, Dhert WJ, Oner FC, Verlaan JJ. Clinical outcome after traumatic spinal fractures in patients with ankylosing spinal disorders compared with control patients. Spine J 2014;14:729-40.

6. Lukasiewicz AM, Bohl DD, Varthi AG, Basques BA,Webb ML,Samuel AM, Grauer JN. Spinal Fracture in Patients With Ankylosing Spondylitis: Cohort Definition, Distribution of Injuries, and Hospital Outcomes. Spine (Phila Pa 1976) 2016;41:191-6.

7. Tavolaro C, Ghaffar S, Zhou H, Nguyen QT, Bellabarba $\mathrm{C}$, Bransford RJ. Is routine MRI of the spine necessary in trauma patients with ankylosing spinal disorders or is a CT scan sufficient? Spine J 2019;19:1331-9. 
8. Koivikko MP, Koskinen SK. MRI of cervical spine injuries complicating ankylosing spondylitis. Skeletal Radiol 2008;37:813-9.

9. Nachtrab O, Cassar-Pullicino VN, Lalam R, Tins B, Tyrrell PN, Singh J. Role of MRI in hip fractures, including stress fractures, occult fractures, avulsion fractures. Eur J Radiol 2012;81:3813-23.

10. Memarsadeghi M, Breitenseher MJ, Schaefer-Prokop C, Weber M, Aldrian S, Gäbler C, Prokop M. Occult scaphoid fractures: comparison of multidetector CT and MR imaging--initial experience. Radiology 2006;240:169-76.

11. Vazan M, Ryang YM, Barz M, Török E, Gempt J, Meyer B. Ankylosing Spinal Disease-Diagnosis and Treatment of Spine Fractures. World Neurosurg. 2019 ;123:e162-70.

12. Caron T, Bransford R, Nguyen Q, Agel J, Chapman J, Bellabarba C. Spine fractures in patients with ankylosing spinal disorders. Spine (Phila Pa 1976) 2010;35:E458-64.

13. Gonzalez-Beicos A, Nuñez DB Jr, Fung AW, Sanchez M, Gahbauer H. Trauma to the ankylotic spine: imaging spectrum of vertebral and soft tissue injuries. Emerg Radiol 2007;14:371-8.

14. Altun I, Yuksel KZ. Ankylosing spondylitis: patterns of spinal injury and treatment outcomes. Asian Spine J 2016;10:655-62.

15. Qiao M, Qian BP, Qiu Y, Mao SH, Wang YH. Radiologic and Pathological Investigation of Pseudarthrosis in Ankylosing Spondylitis: Distinguishing Between Inflammatory and Traumatic Etiology. J Rheumatol 2019;46:259-65.

16. Kim SK, Shin K, Song Y, Lee S, Kim TH. Andersson lesions of whole spine magnetic resonance imaging compared with plain radiography in ankylosing spondylitis. Rheumatol Int 2016;36:1663-70.

17. Ruiz Santiago F, Láinez Ramos-Bossini AJ, Wáng YXJ, López Zúñiga D. The role of radiography in the study of spinal disorders. Quant Imaging Med Surg 2020;10:2322-55.

18. Werner BC, Samartzis D, Shen FH. Spinal Fractures in Patients With Ankylosing Spondylitis: Etiology, Diagnosis, and Management. J Am Acad Orthop Surg 2016;24:241-9.

19. Cha TD, An HS. Cervical spine manifestations in patients with inflammatory arthritides. Nat Rev Rheumatol 2013;9:423-32.

20. Sambrook PN, Geusens P. The epidemiology of osteoporosis and fractures in ankylosing spondylitis. Ther
Adv Musculoskelet Dis 2012;4:287-92.

21. Reinhold M, Knop C, Kneitz C, Disch A. Spine Fractures in Ankylosing Diseases: Recommendations of the Spine Section of the German Society for Orthopaedics and Trauma (DGOU). Global Spine J 2018;8:56S-68S.

22. Tyrrell P, Chowdhury JR. Ankylosing spondylitis and spinal trauma - With an emphasis on the role of radiology. Trauma 2017;19:33-5.

23. Brinckman MA, Chau C, Ross JS. Marrow edema variability in acute spine fractures. Spine J 2015;15:454-60.

24. Charles YP, Buy X, Gangi A, Steib JP. Fracture in ankylosing spondylitis after minor trauma: radiological pitfalls and treatment by percutaneous instrumentation. A case report. Orthop Traumatol Surg Res 2013;99:115-9.

25. Brichko L, Giddey B, Tee J, Niggemeyer L, Fitzgerald M. Cervical spine traumatic epidural haematomas: Incidence and characteristics. Emerg Med Australas 2018;30:359-65.

26. Teunissen FR, Verbeek BM, Cha TD, Schwab JH. Spinal cord injury after traumatic spine fracture in patients with ankylosing spinal disorders. J Neurosurg Spine 2017;27:709-16.

27. Ricart PA, Verma R, Fineberg SJ, Fink KY, Lucas PA, Lo Y, Asprinio DE, Amorosa LF. Post-traumatic cervical spine epidural hematoma: Incidence and risk factors. Injury 2017;48:2529-33.

28. Pizones J, Zúñiga L, Sánchez-Mariscal F, Alvarez P, Gómez-Rice A, Izquierdo E. MRI study of posttraumatic incompetence of posterior ligamentous complex: importance of the supraspinous ligament. Prospective study of 74 traumatic fractures. Eur Spine J 2012;21:2222-31.

29. Henninger B, Kaser V, Ostermann S, Spicher A, Zegg M, Schmid R, Kremser C, Krappinger D. Cervical Disc and Ligamentous Injury in Hyperextension Trauma: MRI and Intraoperative Correlation. J Neuroimaging 2020;30:104-9.

30. Malham GM, Ackland HM, Varma DK, Williamson OD. Traumatic cervical discoligamentous injuries: correlation of magnetic resonance imaging and operative findings. Spine (Phila Pa 1976) 2009;34:2754-9.

Cite this article as: Ren C, Zhu Q, Yuan H. Imaging features of spinal fractures in ankylosing spondylitis and the diagnostic value of different imaging methods. Quant Imaging Med Surg 2021;11(6):2499-2508. doi: 10.21037/qims-20-962 